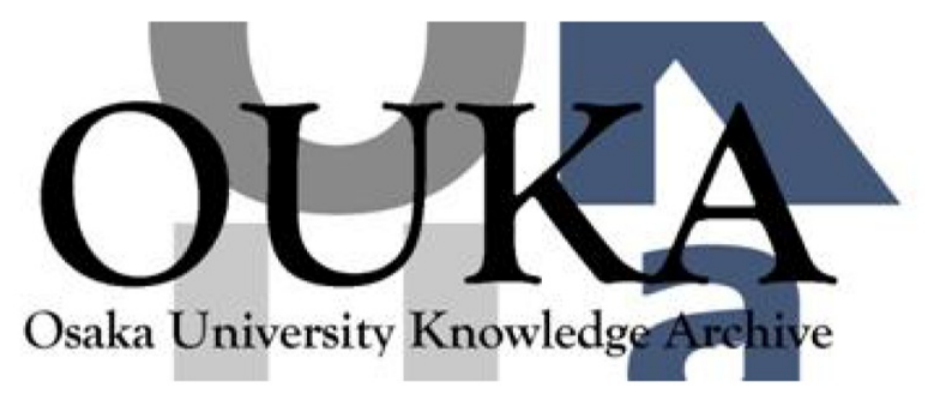

\begin{tabular}{|c|l|}
\hline Title & $\begin{array}{l}\text { Tunable lasing in doped Liquid crystals with } \\
\text { one dimensional periodic structure }\end{array}$ \\
\hline Author(s) & $\begin{array}{l}\text { Ozaki, Masanori; Kasano, Masahiro; Funamoto, } \\
\text { Kazuhiro et al. }\end{array}$ \\
\hline Citation & $\begin{array}{l}\text { Proceedings of SPIE - The International Society } \\
\text { for Optical Eng ineering. 5213 p. 111-p. 122 }\end{array}$ \\
\hline Issue Date & $2004-01-05$ \\
\hline oaire:version & VoR \\
\hline URL & https://hdl. handle. net/11094/76951 \\
\hline rights & \\
\hline Note & \\
\hline
\end{tabular}

Osaka University Knowledge Archive : OUKA

https://ir. Library. osaka-u. ac. jp/

Osaka University 


\title{
Tunable lasing in doped liquid crystals with one dimensional periodic structure
}

\author{
Masanori Ozaki*, Masahiro Kasano, Kazuhiro Funamoto, Ryotaro Ozaki, \\ Tatsunosuke Matsui, and Katsumi Yoshino \\ Department of Electronic Engineering, Graduate School of Engineering, Osaka University \\ Yamada-Oka, Suita, Osaka 565-0871 Japan
}

\begin{abstract}
Various types of tunable lasing in dye-doped liquid crystals with one dimensional periodic structure have been demonstrated. An electrical tuning of lasing wavelength has been demonstrated in a dye-doped chiral smectic liquid crystal mixture with a shor pitch helical structure which is so-called ferroelectric liquid crystal (FLC). Waveguide configuration of FLC laser has also been proposed. also in which the lasing wavelength widely can be tuned upon the electric filed. The electrically tunable lasing has been observed also in a focal conic structure of dye-doped cholesteric liquid crystal. This laser action is based on a helix micro-cavity in focal conic domains. Optically pumped distributed feedback lasing has been proposed in a dye-doped nematic liquid crystal (NLC) waveguide by holographic excitation. in which continuous tuning of the lasing wavelength is performed upon applying electric filed. Electrical tuning of the wavelength of the defect mode lasing in a one-dimensional periodic structure has been demonstrated using a dye-doped NLC as a defect layer in the periodic structure. Lasing wavelength is widely tuned upon applying the electric field. which is due to the refractive index change in the defect layer caused by the field-induced realignment of the NLC molecules.
\end{abstract}

Keywords:

tunable photonic crystal, liquid crystal. laser action. ferroelectric liquid crystal. helical structure. cholesteric liquid crystal. defect mode, holographic excitation

\section{INTRODUCTION}

Photonic crystals (PCs) having a three-dimensional (3-D) ordered structure with a periodicity of optical wavelength have attracted considerable attention from both fundamental and practical points of view. because in such materials novel physical concepts such as photonic band gap have been theoretically predicted and various applications of photonic crystals have been proposed. ${ }^{1.2}$ Especially. the study of stimulated emission in photonic band gap is one of the most attractive subjects, since, in the band gap, a spontaneous emission is inhibited and low-threshold lasers based on photonic crystals are expected. ${ }^{1.3 .6}$ So far intensive studies on one- and two- dimensional band gap materials have been performed. In a one-dimensional periodic structure, the laser action has been expected at the photonic band edge where the photon group velocity approaches zero. ${ }^{7}$ In order to realize the photonic crystal, a large number of intensive studies on a micro-fabrication based on a semiconductor processing technolog $\mathrm{y}^{8-10}$ and a self assembly construction of nanoscale spheres ${ }^{11.12}$ have been carried out.

Liquid crystals (LCs) including chiral molecule have a self-organized helical structure which can be regarded as a 1-D periodic structure. In such systems, there is a so-called stop band in which the light can not propagate, which is considered as a 1-D pseudo-bandgap. Lasing at the band edge has been reported in the cholesteric liquid crystal (CLC). ${ }^{13.14}$ chiral smectic liquid crystal ${ }^{15}$ and polymerized cholesteric liquid crystal. ${ }^{16-19}$ These laser action in the 1-D helical structure of the chiral liquid crystals are interpreted to be based on the band edge of the 1-D photonic band gap in which the photon group velocity is suppressed.?

Chiral smectic liquid crystals with a tilted structure show a ferroelectricity, which are called ferroelectric liquid crystal (FLC). and have an expected potential for the electrooptic applications because of a fast response to the electric field. ${ }^{20}$ 
The FLC also has a helical structure and shows the selective reflection due to the one-dimensional periodic structure as the almost same manner as the CLC. ${ }^{21}$ The helix of FLC can be easily deformed upon applying electric field and its response is fast because of the strong interaction between the spontaneous polarization and electric field. Therefore, a fast modulation of the lasing wavelength upon applying electric field can be expected in a dye-doped FLC.

On the other hand, LCs have a large optical anisotropy and are sensitive to an external stress such as an electric field. Based on such optical anisotropy and field sensitivity. a tunable photonic crystal has been proposed in opal or inverse opal infiltrated with LC. ${ }^{22-27}$ Although opal and inverse opal are simple and inexpensive approach to realize 3-D PC using self-organization of colloidal particles. ${ }^{11.12}$ the introduction of defects into the 3-D periodic structure is a problem that must be resolved. Not only 3-D PCs but also one-dimensional (1-D) PCs are an attractive subject. Although. the 1-D PC does not have a complete PBG, there are plenty of applications using extraordinary dispersion of the photon and localized photonic state in a defect layer. So far, intensive studies on 1-D PC applications have been reported: air-bridge microcavities ${ }^{8}$. the photonic band-edge laser ${ }^{7}$, nonlinear optical diode ${ }^{28}$ and the enhancement of optical nonlinearity. ${ }^{29.30}$ Recently we have introduced a LC layer in dielectric multilayer structure as a defect in I-D PC ${ }^{31}$, in which the wavelength of defect modes were controlled upon applying electric field in a basis of the change in optical length of the defect layer caused by the field-induced molecular reorientation of LC.

In this study, we report various types of tunable lasing in dye-doped LCs with 1-D periodic structure. We successfully demonstrate a lasing wavelength tuning in a wide range upon applying electric field for the first time. In addition, also in the CLC. an electrically tunable laser action is observed in a focal conic structure generated by the electric field application. We also propose two types of laser device based on the combination of LC and periodic structure for the tunable PC. One of them is holographically pumped distributed feedback (DFB) laser using the NLC as an active core layer of the waveguide. Another one is a wavelength tunable laser based on an electrically controllable defect mode in a I-D dielectric periodic structure containing a dye-doped LC as a defect layer. The lasing in 1-D PC with LC defect layer can be tuned in a wide range upon applying electric field.

\section{EXPERIMENTAL}

For the host compound of FLC laser. a multi-component mixture having the chiral smectic $\mathrm{C}$ (SmC*) phase in a wide temperature range including a room temperature was used. As a laser dye doped in the FLC, a Coumarin 500 (Exciton) was used. The concentration of the dye is $0.2 \mathrm{wt} \%$. The sample was filled into sandwich cells of two types of cell configurations; one is a homeotropically aligned cell and the other is a planar cell configuration for waveguide laser. In the homeotropically aligned cell. aluminum foils were used as both spacers determining cell thickness and electrodes. which allows us to apply the electric field parallel to the glass substrates. The cell gap was $50 \mu \mathrm{m}$. The electrodes distance is $2 \mathrm{~mm}$. In order to obtain a homeotropically aligned cell in which the smectic layers are parallel to the substrates, surfaces were coated with a polyimide (Japan Synthetic Rubber, JALS-2021-R2). In the homeotropically aligned cell, the helicoidal axis is perpendicular to the glass substrates. For a planar cell in which the helical axis is parallel to the glass substrates, surfaces of glass substrates were coated with polyimide (JSR, AL1254) and were rubbed unidirectionally. In order to apply an electric field normal to the helical axis, glass plates coated with an In-Sn oxide (ITO) were used as substrates. Thin glass plates were used as spacers. whose thickness was about $150 \mu \mathrm{m}$.

As an excitation source for FLC laser, a second harmonic light of a regenerative amplifier system based on a Ti:sapphire laser (Spectra Physics) was used. The pulse width, wavelength and pulse repetition frequency of the output laser beam were $150 \mathrm{fs}, 400 \mathrm{~nm}$ and $1 \mathrm{kHz}$, respectively. For the homeotropically aligned cell geometry, the excitation laser beam irradiated the sample at an angle of $45^{\circ}$ with respect to the cell plate normal. The illumination area on the sample was about $0.2 \mathrm{~mm}^{2}$. The emission spectra from the dye-doped FLC were measured from the opposite side of the cell. The collecting direction was perpendicular to the cell surface, which is normal to the smectic layers and along the helical axis. On the other hand. for the waveguide laser, the excitation laser beam irradiated the sample perpendicularly to the cell plate and was rectangularly focused using two lenses. concave lens and cylindrical lens, to area of $0.3 \mathrm{~mm}^{2}$. The long axis of the irradiated area on the sample is parallel to the helical axis of the FLC. The emission along the helical axis passed through the spacer glass film and went outside the cell. The spectrum of the light emitted through the glass spacer was measured using a CCD multichannel photodetecto. The collecting direction was parallel to both the cell surface and the helical axis. 
In the electro-tunable laser based on the CLC, the CLC host with left-handed helix was prepared by mixing an optically active agent with a chiral center(S-811. Merck) and a nematic liquid crystal (E44, Merck). The S-811 concentration was $30 \mathrm{wt} \%$ in E44. As a laser dye dopant in the CLC. [2-[2-4-(Dimethylamino)pheny1] etheny1]-6-methy 1-4H-pyran-4ylidene propanedinitrile (DCM) (Exciton) was used. The concentration of the dye was 1 wt $\%$. The sample was filled into a sandwich cell, which consists of two glass plates coated with an ITO. In order to obtain a homogeneously aligned cell. the surfaces were coated with a polyimide(JSR, AL1254) and rubbed. The CLCs in these cells align its director parallel to the glass plates, that is. the helical axis is perpendicular to the glass substrates. As an excitation source for the CLC laser. a second harmonic light of a Q-switched Nd:yturitium-aluminum-garnet laser (Spectra Physics. Qunata-Ray INDI) was used, whose wavelength, pulse width and pulse repetition frequency were $532 \mathrm{~nm} .8 \mathrm{~ns}$, and $10 \mathrm{~Hz}$, respectively. The excitation laser beam irradiated the sample at an angle of about $45^{\circ}$ with respect to the cell plate normal and was focused using a lens to the area of about $0.3 \mathrm{~mm}^{2}$. The emission spectra from CLCs were measured from an opposite side of their cells.

For the holographically pumped DFB laser, E-44 was used as a waveguide material. As a laser dye dopant in the NLC. DCM (Exciton) was used. The concentration of the dye was $0.7 \mathrm{wt} \%, n_{\mathrm{o}}$ and $n_{\mathrm{e}}$ of this mixture are 1.53 and 1.78, respectively (at $632.8 \mathrm{~nm}$ ). The sample was inserted into the sandwiched cell that is composed of two ITO-coated quartz glass substrates. The cell gap was $2.5 \mu \mathrm{m}$. In order to obtain a planarly aligned cell. the surfaces of substrates were coated with a poly (vinyl alcohol) (PVA) and rubbed. The PVA layer also acted as a low index clad layer $\left(n_{\mathrm{PVA}}=1.52\right.$ ). In such a cell structure, the LC layer acts as a core layer of the slab waveguide and the electromagnetic wave can be confined effectively in the LC layer under the total reflection condition. As an excitation source. a third harmonic light of Q-switched Nd:YAG laser ( $\lambda_{\text {ex }}: 355 \mathrm{~nm}$. pulse width:8nm) was used. A rectangular shaped AC voltage of $1 \mathrm{kHz}$ was applied.

The defect mode laser based on the I-D PC with LC defect layer was pumped by a second harmonic light of a Qswitched Nd:YAG laser (Spectra Physics, Quanta-Ray INDI). The illumination area on the sample is about $0.2 \mathrm{~mm}^{2}$. The excitation laser beam irradiated the sample perpendicularly to the cell plate. The emission spectra from the 1-D PC with dye-doped nematic LC are measured from the opposite side of the cell using the CCD multi-channel spectrometer.

\section{RESULTS AND DISCUSSION}

\subsection{Electrical tunability of lasing wavelength in dye-doped FLC}

The lasing characteristics of FLC in the absence of the electric field have been investigated. Figure I shows emission spectra of dye-doped FLC as a function of pump energy. For low pump energy $(1.76 \mu \mathrm{J} / \mathrm{pulse})$. the spectrum is dominated by a broad spontaneous emission and the dip is observed in the broad spectrum. The dip originates from the selective reflection band due to the helix of FLC. As the excitation energy increases, the emission intensity is enhanced. At high excitation energy (10.4 $\mu \mathrm{J} /$ pulse), lasing appears as a sharp peak at the lower energy edge of the dip. The full width at half maximum (FWHM) of the emission peak is less than $0.5 \mathrm{~nm}$.

The laser light emitted from the FLC is circularly polarized and the sense of the polarization is right-hand, which coincides with the helical sense of the FLC used here. In the stop band due to the helical structure. only the circularly polarized light with the same sense as that of the helix

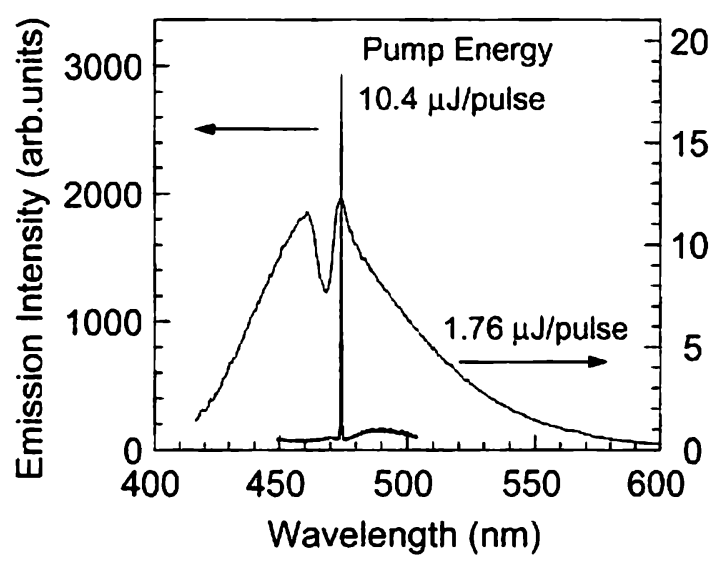

Figure 1: Emission spectra of a dye-doped FLC as a function of pump pulse energy. selectively interacts with the periodic helical structure and is reflected, which is known as selective reflection. Therefore. the result of the polarization dependence measurement strongly supports that the laser action in the dye-doped FLC is based on the band effect in a periodic structure of the FLC helix. 
FLC has a spontaneous polarization $P_{\mathrm{s}}$ which points normal to the molecules and parallel to the smectic layers. When the electric field is applied in the layer, for lower field $\boldsymbol{P}_{\mathrm{s}}$ intends to point along the field direction and FLC molecules starts to reorient toward the direction normal to the, resulting in the deformation of the helix. In the equilibrium state, the deformation of the helix might cause the elongation of the periodicity of the helix. Above the threshold field, all FLC molecules orient to the same direction and the helix is unwound.

The fact that the periodicity of the helical structure of the dye-doped FLC can be controlled upon the field application allows us to expect the electric field tuning of the laser emission wavelength. Figure 2 shows the lasing spectra of the dye-doped FLC at high excitation energy $(24 \mu \mathrm{J} / \mathrm{pulse})$ as a function of the applied electric field. It should be noted that lasing wavelength largely shifts toward longer wavelength with increasing the field, which corresponds to the shift of the selective reflection band. In spite of a low field $(3.5 \mathrm{kV} / \mathrm{cm})$, a wide tuning of the lasing wavelength was achieved. ${ }^{32}$

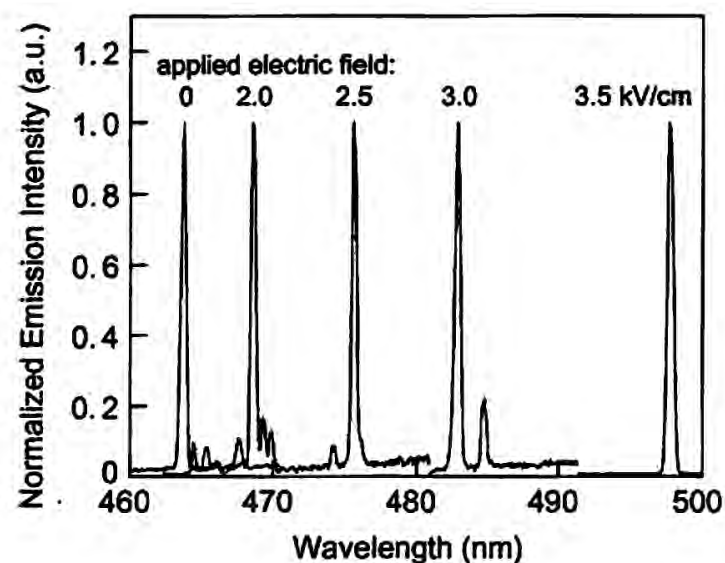

Figure 2: Normalized emission spectra at high excitation energy in a dye-doped FLC as a function of applied electric field.

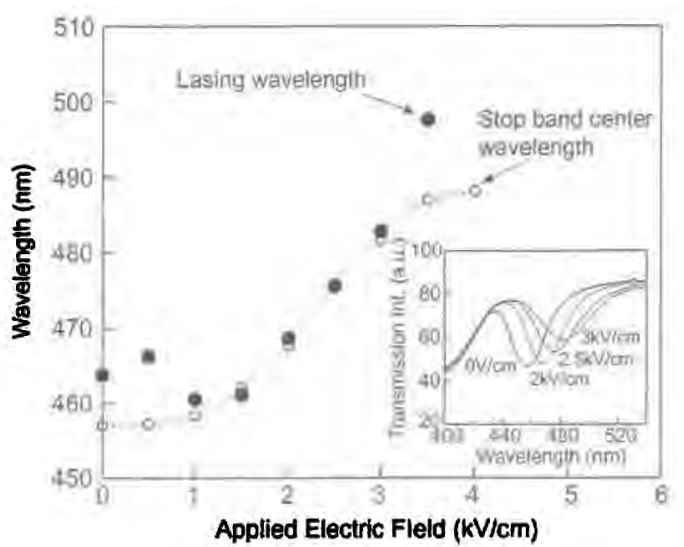

Figure 3: Applied electric field dependences of lasing wavelength and stop band center wavelength of a dyedoped FLC.

Figure 3 shows electric field dependence of the lasing wavelength. The selective reflection center wavelength determined from the transmission spectra (the inset) is also shown as a function of the field. It can be confirmed that the shift of the lasing wavelength is based on the band shift caused by field-induced change in the helix periodicity. It should be noted that for the medium field range the lasing is observed at the center of the stop band.

The electrooptical effects originating from the field-induced deformation of the helix in the FLC have been proposed. ${ }^{33.34}$ Especially, the response of the electrooptical switching based on a small deformation of the helix of a short pitch FLC is as fast as several $\mu \mathrm{s}$ order and the application to an optical communication as well as to a display device has been proposed..$^{35}$ The relaxation times of the helix deformation of FLC is represented by the following equation,

$$
\tau \propto \frac{p^{2} \gamma}{K} .
$$

where $p$ is the helix pitch, $\gamma$ is the rotational viscosity and $K$ is the elastic constant.

According to this relation, the response time is inversely proportional to $p^{2}$, and high frequency modulation of the periodicity of the helix can be expected in a short pitch FLC. In deed, the electrooptic modulation device using a similar compound to the FLC material used in this study has of the order of several $\mu$ s response. Consequently, the fast modulation of the lasing is possible in the dye-doped FLC with a short pitch. 


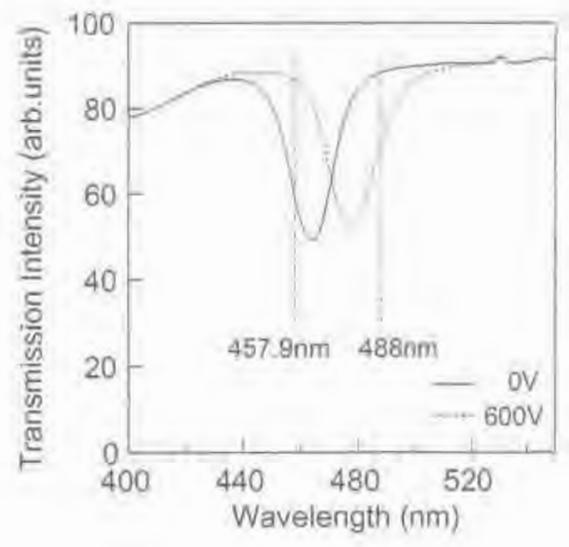

Figure 4: Transmission spectra of FLC as a function of applied voltage.

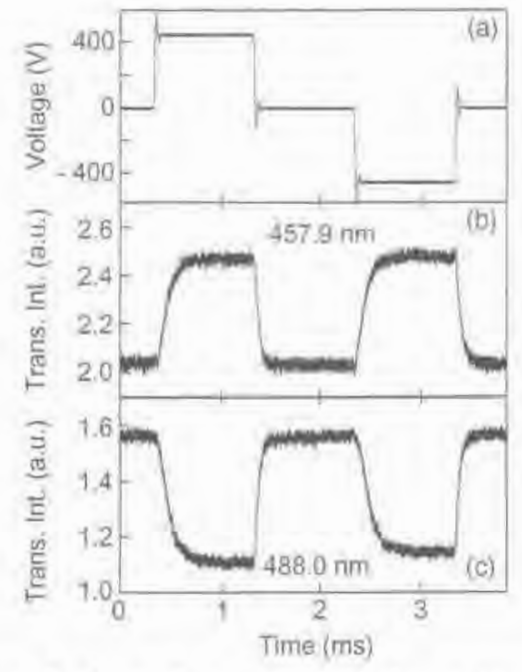

Figure 5: Optical responses to the rectangular voltage at $457.9 \mathrm{~nm}(\mathrm{a})$ and $488 \mathrm{~nm}(\mathrm{~b})$.

In order to investigate the dynamic response of the helix elongation, the transmission intensity change at two wavelengths as shown in Fig.4 was measured upon applying step-wise voltage. Namely, if the stop band in the spectrum shifts toward longer wavelength upon the voltage application, the transmission intensity at $457.9 \mathrm{~nm}$ increases. while that at $488 \mathrm{~nm}$ decreases as shown in Fig.4. Figure 5 shows the transmission change at 457.9 and $488 \mathrm{~nm}$. This shows the response time is about $100 \mu \mathrm{s}$, especially the recovery time at the instance of the voltage removal is less than $100 \mu \mathrm{s}$.

\subsection{Waveguide laser of FLC}

Lasing in the FLC mentioned above has been performed in the cell configuration in which a helical axis is perpendicular to the substrates and laser light is emitted out perpendicularly to the cell surface, as shown in Fig. 6(a). In this configuration, it is not easy to achieve alignment of high quality in a thick cell, so that it is difficult to extend an active region for the lasing. Moreover, a pump beam is absorbed in the vicinity of the interface between liquid crystal and substrate, and doped dye in a bulk is not effectively excited. We design a planar cell configuration of dye-doped FLC for lasing as shown in Fig.6(b) and demonstrate optically pumped lasing in a waveguide. ${ }^{36}$ In this waveguide liquid crystal laser, emission wavelength can be widely controlled by applying electric field.

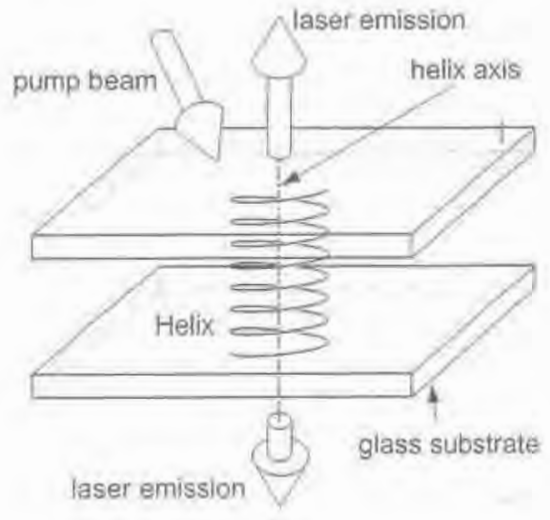

(a)

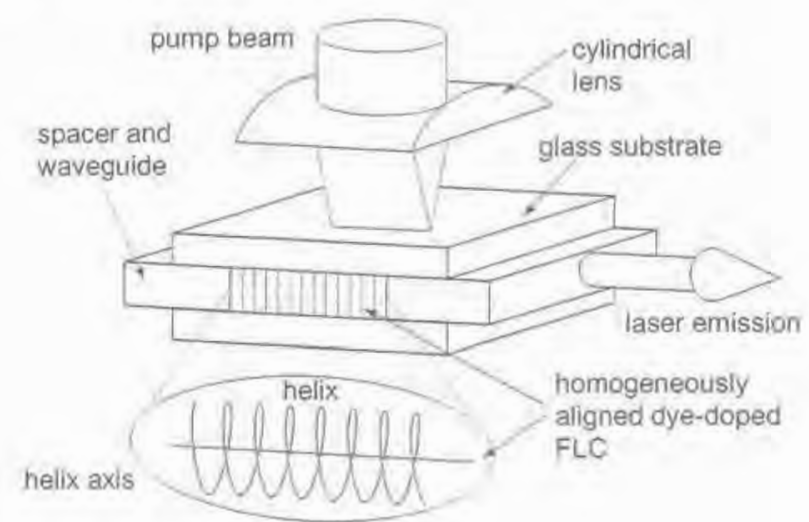

(b)

Figure 6: Cell configurations for the laser action; (a) homeotropically aligned cell, (b) planarly aligned cell for waveguide lasing. 
As the same manner as the case of homeotropically aligned cell configuration, there exists a lasing threshold in the pump energy dependence of the peak intensity and linewidth of the emission spectrum in.the waveguide configuration. At lower excitation energy, the emission intensity increases in proportion to the pump energy. Above the threshold at a pump pulse energy of about 10 $\mu \mathrm{J} /$ pulse, the emission intensity nonlinearly increases. The linewidth of the emission spectrum also drastically decreases above the threshold. These results confirm that lasing occurs above the threshold of the pump energy at the edge of the photonic stop band in the spontaneous emission.

Figure 7 shows laser emission spectra as a function of the voltage applied perpendicularly to the helical axis. As is evident from the figure, the lasing wavelength shifts toward longer wavelength with increasing voltage. In spite of a low field $(2.0 \mathrm{kV} / \mathrm{cm})$, a wide tuning of the lasing wavelength was achieved.

\subsection{Electric field effect of laser action in cholesteric liquid crystal}

Figure 8 shows applied voltage dependence of emission intensity and FWHM of laser action in a dye-doped CLC at high excitation energy. The laser light was emitted in the direction perpendicular to the cell substrates. Above the threshold voltage, the emission intensity is highly suppressed and peak width becomes wider. This indicates that the laser action turns to an amplified stimulated emission. On the other hand, under the electric field, an intense emission with a narrow spectral width has been observed from the side of sandwich cell. This emission parallel to the substrates has never been observed in the absence of the electric filed. Namely, in exchange for the out-of-plane emission, this in-plane emission appears upon applying voltage. There is a threshold pump energy for the intense in-plane emission under the field. Below the threshold, the emission spectrum is broad, while the spectral width is suddenly reduced above the threshold. This means that the in-plane emission is laser action.

In order to clarify the molecular alignment in the cell under the field, a microscopic observation has been carried out. In the absence of the field, a Grandjean texture characteristic to the cholesteric planar alignment was observed. Above the threshold electric field for the exchange from the in-plane lasing to the out-of-plane one, the texture of the microscopic observation changes to the focal conic one which contains many domains with a helix axis not perpendicular to the substrates. Therefore, the in-plane laser emission might be attributed to the laser action from these microcavity in each focal conic domains.

Figure 9(a) shows applied voltage dependence of the in-plane laser emission spectra. As is evident from this figure, the laser emission peak shifts toward longer wavelength as the applied voitage increases. This corresponds to the shift of the 
stop band wavelength, that is, the elongation of the helix in the focal conic domain. As mentioned above, in the FLC, the stop band shift upon applying electric filed can be easily observed. In the CLC, however, the shift of the lasing peak has never been reported so far. This difference between FLC and CLC may originate from the difference of the anchoring strength of the molecules at the surface. In the homeotropically aligned cell of FLC. the anchoring of the molecules at the surface of the substrates is weak so that the molecules close to the surface can move around the helix. On the contrary, in the planarly aligned cell of the CLC, the molecules at the surface are relatively strongly anchored at the substrate and can hardly rotate around the helix. As the result, in the CLC, upon applying electric filed, the helix is deformed and the periodicity can not be smoothly increased, resulting in the disappearance of the stop band and laser action.

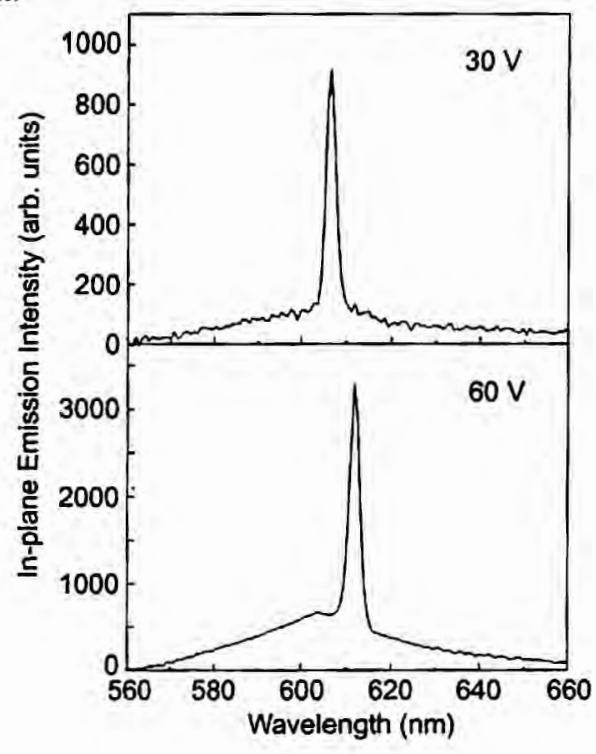

(a)

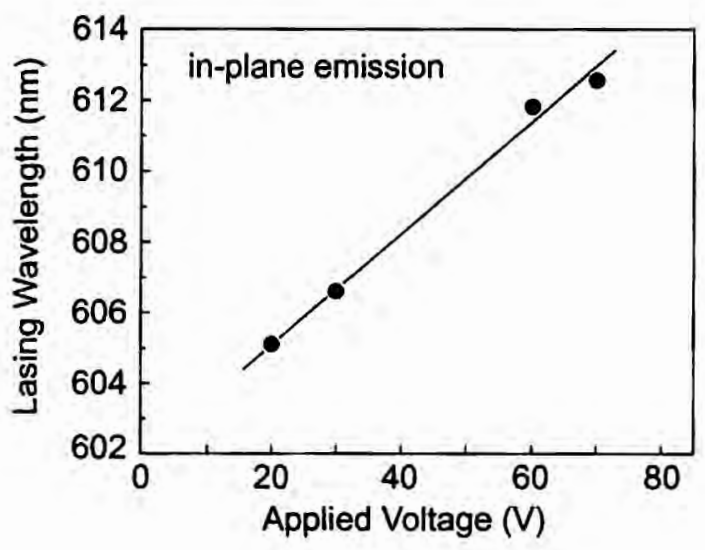

(b)

Figure 9: (a) Emission spectra of in-plane lasing of dye-doped CLC as a function of applied voltage. (b) Applied voltage dependence of the in-plane lasing wavelength of dye-doped CLC.

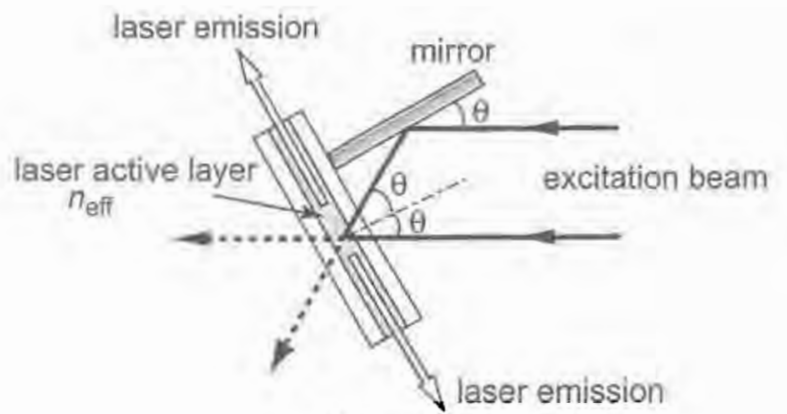

(a)

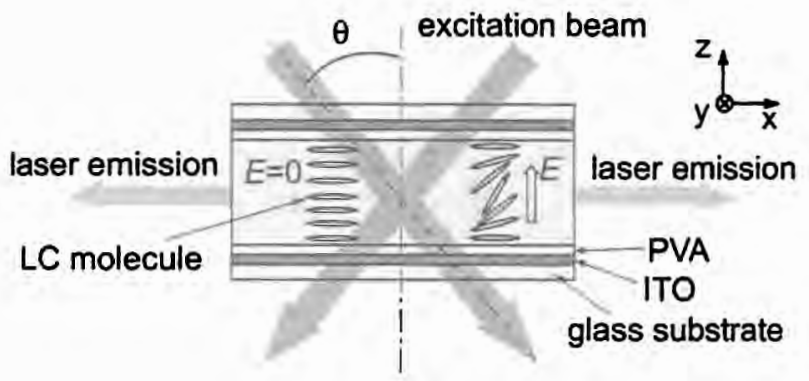

(b)

Figure 10: (a) The Lloyd mirror configuration for the holographic excitation. (b) Schematic explanation of the cell structure for the tunable waveguide laser upon holographic excitation.

\subsection{Electrically tunable lasing in nematic waveguide under holographic excitation}

The distributed feedback (DFB) laser action can be achieved by a transient grating using interference fringes induced by two excitation laser beams (holographic excitation) as shown in Fig.10(a) In this geometry, the lasing wavelength $\lambda_{\mathrm{DFB}}$ upon holographic excitation can be expressed by following equation, 


$$
\lambda_{\mathrm{DFB}}=n_{\mathrm{eff}} \lambda_{\mathrm{ex}} / m \sin \theta,
$$

where $n_{\text {eff }}$ is the effective refractive index of active medium, $\lambda_{\text {ex }}$ is the wavelength of excitation beams, $m$ is the order of diffraction and $\theta$ is the half-angle between two excitation beams. According to this equation, the lasing wavelength can be tuned by changing $\theta$ and/or $n_{\text {eff. }}$ Therefore, if $n_{\text {eff }}$ can be electrically controlled, an electrical tuning of laser emission upon holographic excitation is expected. Based on this concept, we propose an electrical tuning of lasing wavelength using a dye-doped nematic liquid crystal (NLC) as an active laser medium as schematically shown in Fig. 10 (b). ${ }^{37}$ If LC having extraordinary and ordinary refractive indices, $n_{e}$ and $n_{o}$, is used as an active material for the laser medium, the effective refractive $n_{\text {eff }}$ can be electrically controlled due to the field-induced reorientation of LC molecules. Therefore, when a dye-doped nematic LC waveguide is holographically excited, lasing wavelength should be tuned upon changing applied electric filed across the LC layer.

Figure 11 shows the emission spectra of the dye-doped NLC waveguide at various applied voltages. The half angle between two excitation laser beams $\theta$ was fixed to be $65.0^{\circ}$. When the applied voltage $V$ was below $0.7 \mathrm{~V}$, laser emission peak appeared at $599 \mathrm{~nm}$ (peak 1). Above $V=0.8 \mathrm{~V}$, another new peak (peak 2) appeared in longer wavelength. The peak 2 showed a continuous red-shift with increasing the applied voltage. Above $V=1.0 \mathrm{~V}$, the laser emission of peak 2 became multi-mode. Finally, the lasing peak 2 disappeared above $1.4 \mathrm{~V}$. On the other hand, the peak 1 did not shift at all. The intensity of peak 1 decreased gradually, and laser emission peak 1 disappeared above $1.1 \mathrm{~V}$. Applied voltage dependences of the lasing wavelength of peak 1 (open circle) and peak 2 (only main peak, closed circle) are shown in Fig.11(b). The threshold voltage for the appearance of peak 2 is observed at about $0.8 \mathrm{~V}$, which should be associated with the Frederiks transition of the NLC. The electrical tuning of laser action could be performed reversibly.

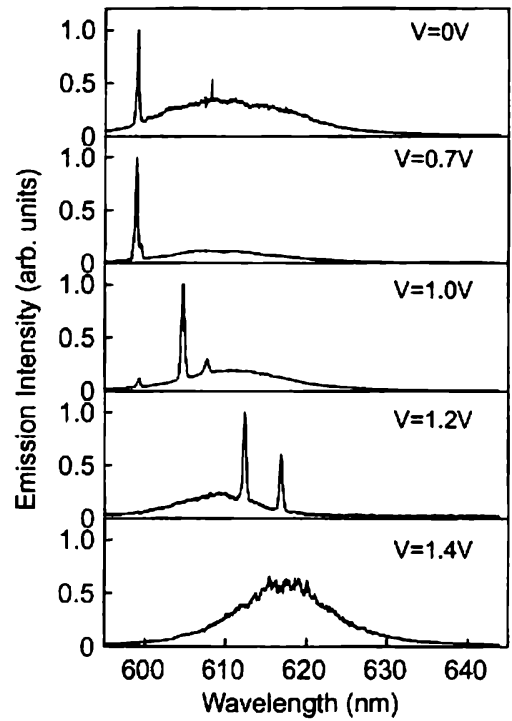

(a)

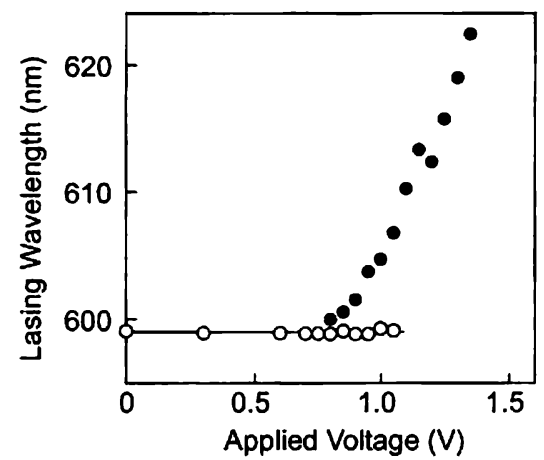

(b)

Figure 11: (a) Emission spectra of the dye-doped NLC waveguide upon holographic excitation as a function of applied voltage. (b) The applied voltage dependence of lasing wavelength of the electrotunable NCL laser.

In the absence of the electric field, the initial LC alignment direction (along $x$-axis) in the active layer lies in the incident plane of two excitation beams and parallel to the substrates. Under such an illumination condition, both transverse electric (TE) and transverse magnetic (TM) -guided modes in the NLC core layer feels the ordinary refractive index $n_{0}$ of the NLC. When the voltage is applied, the reorientation of NLC molecules takes place in the $x z$-plane, so that the effective refractive index $n_{\text {eff }}$ for TM-guided mode increases gradually with the field-induced reorientation of the NLC and the lasing wavelength associated with TM-guided mode should shift to longer wavelength. Consequently, the red- 
shift of the lasing peak 2 might be attributed to TM-guided mode. With the increase in $n_{\text {eff }}$, the effective thickness of the core layer also increases and higher order guided mode should appear, resulting in the appearance of multi-mode emission in peak 2 about $1.0 \mathrm{~V}$.

On the other hand. TE-guided mode feels $n_{\mathrm{o}}$ of the NLC regardless of the applied voltage, so that no change in lasing wavelength of peak 1 should be attributed to TE-guided mode. Although no change of lasing wavelength was observed. the lasing intensity of peak 1 was reduced and peak $I$ disappeared at more than about $1.1 \mathrm{~V}$. This is attributed to the redshift of the photoluminescence (PL) spectrum of the doped laser dye caused by the increase in the effective refractive index of the NLC core.

\subsection{Tunable defect mode lasing in a periodic structure containing $\mathrm{LC}$ layer as a defect}

We have introduced a LC layer in a I-D PC as a defect, in which the wavelength of defect modes were controlled upon applying electric field in a basis of the change in optical length of the defect layer caused by the field-induced molecular reorientation of LC. ${ }^{31}$ We here propose a wavelength tunable laser based on an electrically controllable defect mode in a 1-D dielectric periodic structure containing a dye-doped LC as a defect layer. ${ }^{38}$

Figure 12 shows the I-D PC with a LC defect. A dielectric multilayer consisting of an alternating stack of $\mathrm{SiO}_{2}$ and $\mathrm{TiO}_{2}$ layers deposited on an In-Sn oxide (ITO) coated glass substrate is used as the I-D PC. The center wavelength of the photonic band is adjusted to be $600 \mathrm{~nm}$ by setting the optical thickness of both $\mathrm{SiO}_{2}$ and $\mathrm{TiO}_{2}$ to be one-quarter of 600 $\mathrm{nm}$. The refractive indices of $\mathrm{SiO}_{2}$ and $\mathrm{TiO}_{2}$ are 1.46 and 2.35, respectively, and the thickness of $\mathrm{SiO}_{2}$ and $\mathrm{TiO}_{2}$ layers are $103 \mathrm{~nm}$ and $64 \mathrm{~nm}$, respectively. The number of $\mathrm{SiO}_{2}-\mathrm{TiO}_{2}$ pairs on each substrate is 5 . The top surface of the dielectric multilayer is coated with a polyimide (JSR, AL 1254) and unidirectionally rubbed along $y$-axis in Fig.12.

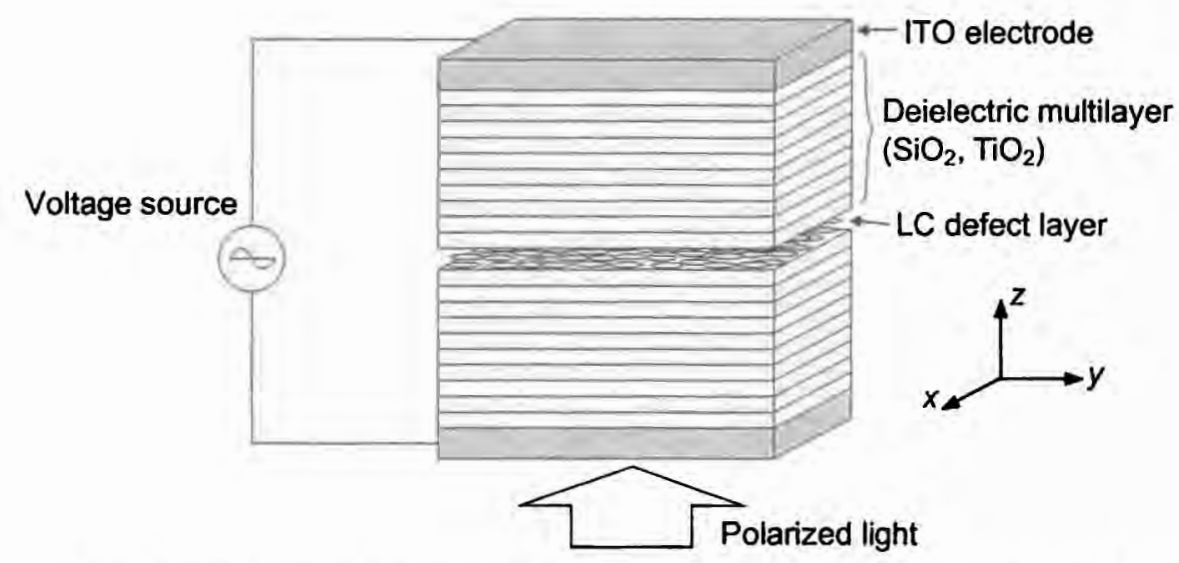

Figure 12: Schematic representation of 1-D PC containing LC layer as a defect.

In order to introduce the defect layer, a dye-doped nematic liquid crystal (Merck. E47) was sandwiched between substrates with dielectric multilayer using $2 \mu \mathrm{m}$ spacers. The refractive index anisotropy $\Delta n$ of E47 is 0.209 at room temperature. In the absence of an electric field, the long molecular axis of the LC is aligned parallel to the substrates ( $y$ axis). As a laser dye doped in the LC, DCM (Exciton) is used. The concentration of the dye is $0.5 \mathrm{wt} . \%$.

In order to investigate the characteristics of defect mode, the transmission spectrum of the linearly polarized light propagating along the $z$-axis was measured from the opposite side of the cell using a CCD multi-channel spectrometer having a spectral resolution of $3 \mathrm{~nm}$. Figure 13 shows the voltage dependence of transmission spectra for the incident light polarized along the $y$-axis which corresponds to the rubbing direction and the initial orientation direction of the LC molecules in the defect layer as shown in Fig.12. A rectangular wave voltage of $1 \mathrm{kHz}$ was applied between ITO layers to change the molecular alignment of the LC in the defect layer. The solid and dashed lines correspond to spectra at $0 \mathrm{~V}$ and $1.2 \mathrm{~V}$, respectively. The peaks of the defect modes shift to shorter wavelengths upon applying voltage. This peak 
shift originates from the decrease in the optical length of the defect layer caused by the field-induced reorientation of the LC molecules. Consequently, we confirmed that the wavelength of the defect mode in 1-D PC with the LC layer as a defect can be controlled upon applying voltage.

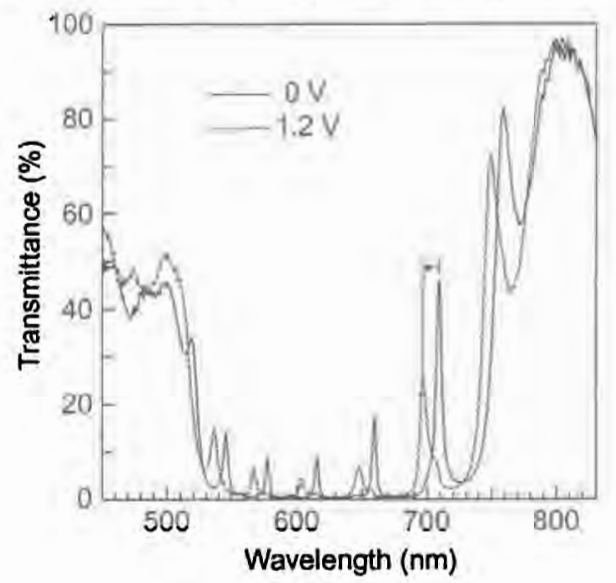

Figure 13: Transmission spectra of 1-D PC with a LC defect as a function of applied voltage.

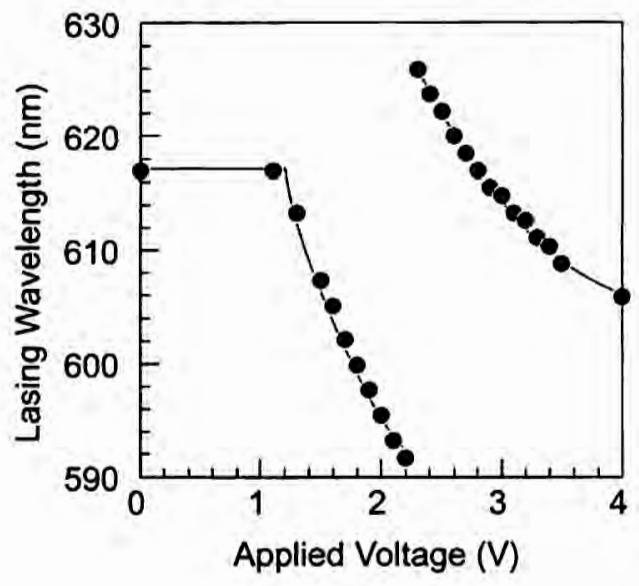

(a)

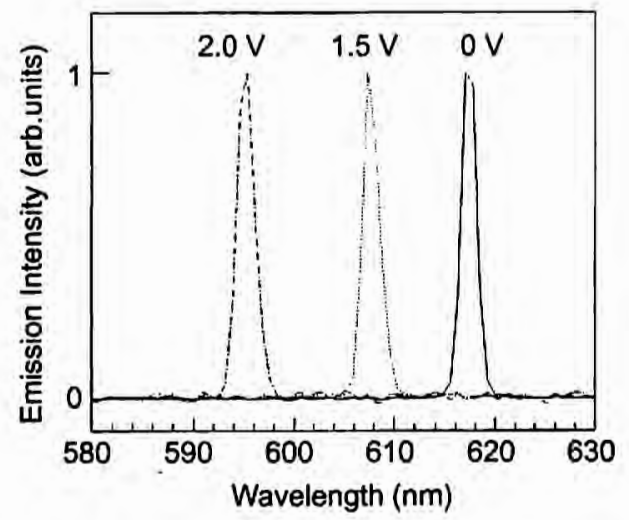

Figure 14: Emission spectra of 1-D PC with the dyedoped NLC defect as a function of applied voltage.

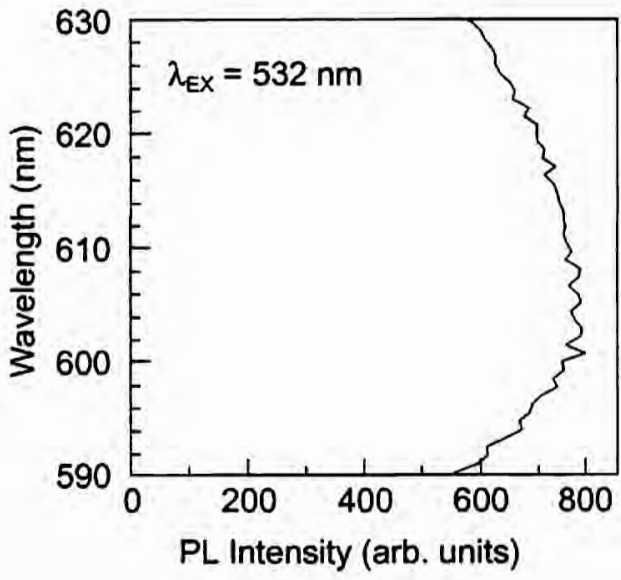

(b)

Figure 15: (a) Voltage dependence of the defect-mode lasing wavelength in the 1-D PC with dye-doped NLC defect. (b) Photoluminescence spectrum of the dye-doped NLC without I-D PC.

Figure 14 shows the lasing spectra of 1-D PC with the DCM-doped LC as a function of the applied voltage. A sharp emission peak appears above the threshold at pump pulse energy of about $3 \mu \mathrm{J}$. The FWHM of the lasing peak is about 3 $\mathrm{nm}$, which is limited by the spectral resolution of the CCD spectrometer used in this experiment. The FWHM of this emission peak is narrower than that of the transmission peak of the defect mode $(10 \mathrm{~nm})$ shown in Fig. 13. This indicates that the emission peak in the band is not the spontaneous emission of DCM passing through the narrow band window of a defect mode.

It should be noted that lasing peak shifts toward shorter wavelengths with increasing voltage as the same manner as the defect modes shift shown in Fig. 13. The wavelength shift of the lasing peak is about $25 \mathrm{~nm}$ even upon applying low 
voltage. Figure 15(a) shows voltage dependence of the lasing peak wavelength. As is evident from Fig. 15(a). a threshold voltage exists for the peak shift and the lasing wavelength shifts toward shorter wavelengths above the threshold of $1.1 \mathrm{~V}$. This is associated with Frederiks transition of the LC in the defect layer. In Fig. 15(a), the discontinuous change of the lasing wavelength is observed around $2.1 \mathrm{~V}$. This means that the defect mode at shorter wavelengths go out of the wavelength range of the high emission efficiency of DCM (Fig.15(b)) above $2.1 \mathrm{~V}$. On the other hand, the defect mode peak at the longer wavelengths appears in the lasing range above $2.3 \mathrm{~V}$.

\section{SUMMARY}

Tunable lasing in dye-doped liquid crystals with one dimensional periodic structure was proposed. We used two approaches to realize the tunable laser; that is, to use a self organization characteristics of chiral LC and the combination of LC and periodic structure. The former is the dye-doped FLC and CLC, and the latter is the 1-D PC containing dyedoped NLC as a defect and the holographically pumped DFB laser having dye-doped NLC core layer of the waveguide. In all cases, the electrically tunable laser action was demonstrated.

This work is supported by a Grant-in-Aid for Scientific Research from the Japan Ministry of Education, Culture, Sports, Science and Technology (14350165). We would like to show our acknowledgements to Professor W.Haase for providing the short pitch FLC materials and to Merck KGaA for providing the photo-polymerizable CLC materials.

\section{REFERENCES}

1. E. Yablonovitch, Phys. Rev. Lett. 58, 2059, 1987.

2. S. John, Phys. Rev. Lett. 58, 2486, 1987.

3. J. D. Joannopoulos, R. D. Meade, J. N. Winn: Photonic crystals, Molding the Flow of Light, Princeton Univ. Press, Princeton, NJ, 1995.

4. J. Martorell, N. M. Lawandy, Phys. Rev. Lett. 6, 1877, 1990.

5. K.Yoshino, S.B.Lee, S.Tatsuhara, Y.Kawagishi, M.Ozaki, A.A.Zakhidov, Appl. Phys. Lett., 73, 3506, 1998.

6. K.Yoshino, S.Tatsuhara, Y.Kawagishi, M.Ozaki, A.A.Zakhidov, Z.V.Vardeny, Appl. Phys. Lett., 74, 2590, 1999.

7. J. P. Dowling, M. Scalora, M. J. Bloemer, C. M. Bowden, J. Appl. Phys. 75, 1896, 1994.

8. J. S. Foresi, P. R. Villeneuve, J. Ferrera, E. R. Thoen, G. Steinmeyer, S. Fan, J. D. Joannopoulos, L. C. Kimerling, H. I. Smith and E. P. Ippen, Nature 390, 143, 1997.

9. O. Painter, R.K. Lee, A. Scherer, A. Yariv, J.D. O’Brien, P.D. Dapkus and I. Kim, Science 284, 1819, 1999.

10. S. Noda, K. Tomoda, N. Yamamoto and A. Chutinan, Science 289, 604, 2000.

11. K. Yoshino, K. Tada, M. Ozaki, A. A. Zakhidov, R. H. Baughman, Jpn. J. App. Phys., 36, L714, 1997.

12. Y.A.Vlasov, K.Luterova, I.Pelant, B.Honerlage, V.N.Astratov, Appl.Phys.Lett., 71, 1616, 1997.

13. V. I. Kopp, B. Fan, H. K. Vithana, A. Z. Genack, Opt. Lett. 23, 1707, 1998

14. B. Taheri, A. F. Munoz, P. Palffy-Muhoray and R. Twieg, Mol. Cryst. Liq. Cryst. 358, 73, 2001.

15. M. Ozaki, M. Kasano, D. Ganzke, W. Haase, K. Yoshino, Adv. Mater. 14, 306, 2002.

16. H. Finkelmann, S. T. Kim, A. Munoz, P. Palffy-Muhoray, B. Taheri, Adv. Mater. 13, 1069. 2001.

17. J. Schmidtke, W. Stille, H. Finkelmann, S.T. Kim, Adv.Mater. 14, 746, 2002.

18. T. Matsui, R. Ozaki, K. Funamoto, M. Ozaki and K. Yoshino, Appl.Phys.Lett., 81, 3741, 2002.

19. M. Ozaki, R. Ozaki, T. Matsui and K.Yoshino, Jpn.J.Appl.Phys. 42, L472, 2003.

20. J. W.Goodby, R. Blinc, N.A. Clark, S.T. Lagerwall, M.A. Osipov, S.A. Pikin, T. Sakurai, K.Yoshino, B. Zeks, Ferroelectric Liquid Crystals, Gordon and Breach Science Publishers, Philadelphia, 1991.

21. K. Hori, Mol.Cryst.Liq.Cryst. 82, L13, 1982.

22. K. Yoshino, S. Satoh, Y. Shimoda, Y. Kawagishi, K. Nakayama and M. Ozaki, Jpn. J. Appl. Phys. 38, L961, 1999.

23. K. Yoshino. Y. Shimoda, Y. Kawagishi, K. Nakayama and M. Ozaki, Appl. Phys. Lett. 75, 932, 1999.

24. K. Busch and S. John, Phys. Rev. Lett. 83, 967, 1999.

25. Y. Shimoda, M. Ozaki and K. Yoshino, Appl. Phys. Lett. 79, 3627, 2001.

26. D. Kang, J. E. Maclennan, N. A. Clark, A. A. Zakhidov and R. H. Baughman, Phys. Rev. Lett. 86, 4052, 2001.

27. M. Ozaki. Y. Shimoda, M. Kasano and K.Yoshino, Adv. Mater. 14, 514. 2002.

28. M. D. Tocci, M. J. Bloemer, M. Scalora, J. P. Dowling and C. M. Bowden, Appl. Phys. Lett. 66, 2324, 1995. 
29. T. Hattori, N. Tsurumachi and H. Nakatsuka, J. Opt. Soc. Am. 14, 348, 1997

30. Y. Dumeige, P. Vidakovic, S. Sauvage, I. Sgnes and J. A. Levenson, Appl. Phys. Lett. 78, 3021, 2001.

31. R. Ozaki, T. Matsui, M. Ozaki and K. Yoshino, Jpn. J. App. Phys. 41, L1482, 2002.

32. M. Ozaki, M. Kasano, D. Ganzke, W. Haase, K. Yoshino, Adv. Mater. 15, 974, 2003.

33. K. Yoshino, K.G. Balakrishnan, T. Uemoto, Y. Iwasaki, Y. Inuishi, Jpn.J.Appl.Pjys. 17, 597, 1978.

34. L. A.Beresnev, V.G. Chigrinov, D.I. Dergachaev, E.P. Pozhidaev, J. Funfschilling and M. Schadt, Liq.Cryst. 5, $1171,1989$.

35. E. Pozhidaev, S. Pikin, D. Ganzke, S. Shevtchenko, W. Haase, Ferroelectrics 246, 235, 2000.

36. M. Kasano, M. Ozaki, D. Ganzke, W. Haase and K. Yoshino, Appl.Phys.Lett., 82, 4026, 2003.

37. T. Matsui, M. Ozaki and K. Yoshino, Appl. Phys. Lett., 83, 2003.

38. R. Ozaki, T. Matsui, M. Ozaki and K. Yoshino, Appl.Phys.Lett. 82, 593-3595, 2003.

39. R. Ozaki, M. Ozaki and K. Yoshino, Jpn.J.Appl.Phys. 42, L669, 2003. 